Convergences francophones 6.3 (2020): 41-57

http://mrujs.mtroyal.ca/index.php/cf/index

\title{
Le verso de la p(l)age de Camus: intertextualité militante dans Meursault, contre-enquête
}

\author{
Zvezdana Ostojic \\ John Hopkins University, États-Unis
}

«Aujourd'hui, M'ma est encore vivante.»: voici la phrase qui ouvre Meursault, contre-enquête (2013/2014), écrit par l'écrivain et journaliste algérien Kamel Daoud (13). Le lecteur un tant soit peu attentif devinera immédiatement le jeu intertextuel en question : il s'agit bien évidemment de l'inversion du fameux incipit de L'Étranger de Camus. ${ }^{1}$ Dès le début, le roman de Daoud s'adresse à un public bien ciblé de lecteurs qui connaissent leurs classiques du $20^{\mathrm{e}}$ siècle ${ }^{2}$, ou qui du moins prévoient de les lire en parallèle de leurs nouvelles découvertes parmi la littérature contemporaine. Qu'ils soient amateurs confirmés de L'Étranger ou qu'ils soient indifférents voire hostiles envers l'œuvre de Camus, les lecteurs seront surpris de voir que Daoud ose réécrire l'illustre œuvre canonique de 1942 en se servant de structures empruntées à ce genre réputé «mineur» qu'est le roman policier. Longtemps considéré comme étant de moindre qualité littéraire et intellectuelle que la littérature consacrée, le roman policier continue encore aujourd'hui sa lutte pour la légitimité et la pleine reconnaissance institutionnelle. ${ }^{3}$

Tout comme L'Étranger de Camus, Meursault, contre-enquête est raconté à la première personne par un narrateur bavard qui s'appelle Haroun. Assis dans un bar et imbibé d'alcool, il est abattu par le chagrin causé par le meurtre de son frère Moussa, ${ }^{4} 1$ ' « Arabe » tué par Meursault sur la plage en Algérie un jour d'été dans les années trente. Quelques décennies après la publication de L'Étranger, commence la contre-enquête que mène l'interlocuteur d'Haroun, un universitaire anonyme qui ne prendra jamais la parole, et qui, d'après ce qui nous est

\footnotetext{
${ }^{1}$ « Aujourd'hui, maman est morte. Ou peut-être hier, je ne sais pas. » (Camus 1127).

${ }^{2}$ Dans son article « 'L'Étranger', roman solaire » Roland Barthes écrit : «L'Étranger est sans doute le premier roman classique de l'après-guerre (j'entends premier, pas seulement en date, mais en qualité !) » (398).

${ }^{3}$ Voir à ce sujet Uri Eisenzweig, « Quand le policier devint genre » et G.K. Chesterton, « Défense des romans policiers » dans Autopsies du roman policier, et Claire Gorrara, «French crime fiction : from genre mineur to patrimoine culturel ».

${ }^{4}$ Plusieurs critiques ont souligné l'importance de l'onomastique de la dénomination de l'Arabe tué par Meursault. Les uns ont relevé la prononciation du nom Moussa qui ressemble à la distorsion vocale de Meursault (Kaplan), alors que d'autres ont insisté sur la résonance islamique et prophétique du nom Moussa (la variante arabe de Moïse) qui est un des grands prophètes dans le texte coranique (Belhabib, Roseau, Isaac, Brozgal). Le rapport entre Moussa et Moïse est évoqué à maintes reprises à travers le roman : «Qui sait quel fleuve l'a porté jusqu'à la mer qu'il devait traverser à pied, seul, sans peuple, sans bâton miraculeux ? ; « Moussa était donc un dieu sobre et peu bavard, rendu géant par une barbe fournie et des bras capables de tordre le cou au soldat de n'importe quel pharaon antique. »; « Mon frère Moussa était capable d'ouvrir la mer en deux et il est mort dans l'insignifiance, tel un vulgaire figurant, sur une plage aujourd'hui disparue, tout près de flots qui auraient dû le rendre célèbre pour toujours! » (Daoud 14, 19, 20).
} 
communiqué par l'unique énonciateur du livre, Haroun, tente de restituer la biographie de la victime et de retrouver son corps disparu.

Le fardeau sisyphéen dont Haroun souhaite se débarrasser en participant à cette enquête est le statut de victime, ce qui permettrait l'accès à la parole et à l'écriture à ceux et celles qui sont exclus du discours dominant, ayant subi une narration racontée du point de vue du colonisateur. ${ }^{5}$ Partant de cette hypothèse, nous étudierons la spécificité de l'affrontement intertextuel qu'entretient le texte de Daoud avec celui de Camus. En fournissant « l'autre côté » de l'histoire, autrement dit en élucidant la partie de celle-ci que L'Étranger a laissée dans l'ombre, la contre-enquête de Daoud décompose et recompose l'œuvre de Camus. En même temps, il écrit son texte au verso de la page de Camus de manière à créer un reflet dans le miroir, reflet qui, sans être directement inversé, paraît légèrement anamorphe, comme sous une loupe dont les bords déforment l'objet visé. Ce faisant, Daoud déstabilise la position du lecteur qui ne parvient jamais à une solution cohérente sinon définitive du crime, contrairement à ce que propose d'ordinaire au lecteur le roman policier à énigme. De plus, depuis sa position doublement marginale (celle d'un auteur francophone algérien qui adopte les codes du roman policier afin de reprendre une œuvre majeure), Daoud dénude les aspects métatextuels de la création littéraire en général, et par extension éclaire les mécanismes à l'œuvre chez Camus. Il parvient ainsi à subvertir les hiérarchies et à désacraliser l'œuvre canonique. Dans ce combat intertextuel, l'écriture et le crime ne feront qu'un : le corps du texte est comparé au corps-cadavre, le meurtre incite à la création littéraire et les mots «écrivain » et «tueur» deviennent des synonymes. Voilà les stratégies qui permettent à Daoud, à l'instar d'Edipe, de mettre à mort symboliquement son patrimoine ${ }^{6}$ afin de pouvoir raconter sa propre version de l'histoire.

\section{À la recherche du corps perdu}

En dépit des efforts de «l'inspecteur-universitaire », la quête pour le corps disparu de l'Arabe qu'a tué Meursault, finira par échouer, le corps de la victime n'existant in fine qu'à l'intérieur de l'énonciation peu fiable ${ }^{7} \mathrm{~d}$ 'un narrateur à moitié

\footnotetext{
${ }^{5}$ « J'ai tant de fois souhaité tuer Moussa après sa mort, pour me débarrasser de son cadavre, pour retrouver la tendresse perdue de M'ma, pour récupérer mon corps et mes sens... C'est ton héros qui tue, c'est moi qui éprouve la culpabilité » (Daoud 57).

${ }^{6}$ Le texte de Camus est devenu un véritable lieu de mémoire ou patrimoine collectif que Pierre Nora définit comme « toute unité significative, d'ordre matériel ou idéel, dont la volonté des hommes ou le travail du temps a fait un élément symbolique du patrimoine mémoriel d'une quelconque communauté » (20).

${ }^{7}$ Haroun est tout au long du récit représenté comme un narrateur mythomane : « Contrairement à moi, qui, à force de ressasser cette histoire, ne m'en souviens presque plus. »; « Mais là, je te mens, comme je me suis menti à moi-même pendant longtemps. »; " Je ne sais ce qu'il m'a pris, mais quand l'imam m'a demandé quelle était la cause de mon chagrin, j'ai accusé un gamin de m'avoir battu. C'était, je crois, mon premier mensonge... Car, à partir de ce moment-là, je devins rusé et fourbe, je me mis à grandir » (Daoud 11, 20, 70).
} 
ivre. Ce n'est pas pour rien que le bar où ce dernier fabule est surnommé le « Titanic ». ${ }^{8}$ Ici le mystère n'est pas l'identité du meurtrier, ni de ses motivations ; l'accent est mis sur la victime, alors que le suspense s'accroît à chaque page : Qu'est-il arrivé à Moussa et qu'est-il advenu de son corps ? En donnant à la victime anonyme et sans visage de L'Étranger un nom propre, Haroun ressuscite dans et par la langue le corps disparu ; aussi le texte de la contre-enquête de Daoud donnet-il chair à l'Arabe à qui L'Étranger avait ôté le corps, et qui vient remplir dorénavant le tombeau laissé vide par le texte de Camus : "Sans m'en rendre compte, et des années avant que je n'apprenne à lire, je refusais l'absurdité de sa mort et j'avais besoin d'une histoire pour lui donner un linceul » (Daoud 31). La narration est cet espace discursif qui permet de redonner corps à son frère, car sans l'histoire que raconte Haroun, le frère sans sépulture aurait été oublié à jamais : « M'ma a toujours refusé de me décrire ses traits, son caractère, de lui donner un corps ou de me raconter le moindre souvenir » (94). Ainsi, le narrateur qui nomme celui qui dans L'Étranger a été effacé (de la page comme de la plage), se transforme en gardien du corps-cadavre qu'il porte, tel Sisyphe, ${ }^{9}$ vers tel sommet : ${ }^{10}$ " Je me sentais à la fois coupable d'être vivant mais aussi responsable d'une vie qui n'était pas la mienne! Gardien, assasse, comme mon père, veilleur d'un autre corps. » (44-45). Ce n'est pas pour rien que le corps de Moussa, effacé dans le texte de $L$ 'Étranger, est comparé au parchemin ${ }^{11}$ et sa peau au papier :

cette façon qu'avait mon frère de jouer avec son couteau et de me montrer ses tatouages. 'Echedda fi Allah' ('Dieu est mon soutien'). 'Marche ou crève', sur son épaule droite. 'Tais-toi' avec, dessiné sur son avant-bras gauche, un cœur brisé. C'est le seul livre écrit par Moussa. Plus court qu'un dernier soupir, se résumant à trois phrases sur le plus ancien papier du monde, sa propre peau. Je me souviens de ses tatouages comme d'autres de leur premier livre d'images (30)

Au cours du récit, le lecteur apprend que comme Meursault, Haroun est lui-même assassin ; quelques jours après la proclamation de l'indépendance de l'Algérie, il aurait tué un Français nommé Joseph Larquais. Meurtriers tous deux, Haroun et Meursault sont aussi tous deux narrateurs autoritaires, le lecteur étant appelé à jouer dans les deux cas le rôle du témoin complice de crimes sans motifs apparents. Or le lecteur assume ce rôle à contre-cœur puisque tout au contraire des romans policiers à énigme qui placent le lecteur du côté de l'enquêteur, autrement dit du côté de l'ordre supposé légitime et du «bien », la contre-enquête de Daoud place le lecteur du côté d'un meurtrier. Vu que l'unique source des informations est le meurtrier lui-même, le récit risque de prendre le lecteur au piège dans l'univers clos

\footnotetext{
${ }^{8}$ Ce cadre n'est pas sans rappeler un autre texte de Camus, notamment La Chute.

${ }^{9}$ Ceci est un clin d'œil à une autre œuvre de Camus - Le Mythe de Sisyphe, second volet du cycle sur l'absurde de Camus, publié quelques mois après L'Etranger.

${ }^{10}$ «l'absurdité de ma condition [qui] consistait à pousser un cadavre vers le sommet du mont avant qu'il ne dégringole à nouveau, et cela sans fin » (Daoud 59).

${ }_{11}$ «Regarde sa peau, on dirait un parchemin » (Daoud 35).
} 
et claustrophobique du narrateur en train de mener son «monologue prétentieux » (56).

Le monologisme apparent qu'assure le meurtrier rétrécit la diégèse à tel point que le lecteur a l'impression d'être à l'intérieur de la tête dérangée de Haroun, où tout est écrit au préalable : «Vas-y, remets-toi donc à lire, même si tout est écrit dans ma tête » (17). Le corps du récit n'est rien d'autre que le prolongement du corps du narrateur, alors que le texte " écrit dans sa tête » tente de faire exhumer le cadavre que $L$ 'Étranger a si savamment dissimulé.

\section{L’inversion difforme et la réécriture postcoloniale}

Comme tout remake postcolonial, ${ }^{12}$ le livre de Daoud inverse la perspective adoptée dans L'Étranger, car l'histoire y est censée être racontée non plus du point de vue du protagoniste européen, mais du point de vue du colonisé. À en croire Haroun, Meursault, contre-enquête commencerait par la fin et s'écrirait, comme il se doit, de droite à gauche : «C'est simple : cette histoire devrait être réécrite, dans la même langue, mais de droite à gauche. C'est-à-dire en commençant par le corps encore vivant, les ruelles qui ont mené à sa fin, le prénom de l'Arabe, jusqu'à sa rencontre avec la balle.» (Daoud 16). Toutefois, plus qu'il ne s'oppose diamétralement à son prédécesseur, cette œuvre en miroir se présente comme quelque peu difforme et anamorphe. Bien que le narrateur promette une histoire racontée « de droite à gauche » comme dans la langue arabe qui aurait ressuscité « le corps encore vivant » de la victime, il n'éclaire pas pleinement la vie et la mort de son frère, et finit par raconter l'histoire d'un deuxième crime, ce meurtre qui transforme Haroun en un double de Meursault et le rapproche plus de l'assassin que de la victime (16). C'est bien une question de sang car Haroun prolonge et complète «l'œuvre » de Meursault : «J'ai appuyé sur la détente, j'ai tiré deux fois. Deux balles... Au total, cela fait sept, pensai-je sur le champ, absurdement. (Sauf que les cinq premières, celles qui avaient tué Moussa, avaient été tirées vingt ans auparavant) » (85). En reproduisant la structure du texte de Camus, ${ }^{13}$ plutôt que d'aboutir nous semble-t-il à la création d'un palimpseste hypertextuel qui symbolise un geste émulateur, Daoud écrit son texte avec du sang et communique avec le texte de Camus d'une manière plus violente. De toute évidence, Meursault, contre-enquête se rapproche de ce que Genette appelle des "continuations meurtrières » où l'hypertexte est « à la fois une continuation et une réfutation » de

\footnotetext{
${ }^{12}$ Voir à ce sujet Lia Brozgal, «The Critical Pulse of the Contre-enquête: Kamel Daoud on the Maghrebi Novel in French »: «In the case of the 'postcolonial remake' (a work that reconfigures or reimagines the narratives of colonial, or Western, texts), the critical stakes of appropriation are necessarily politically charged, insofar as the reprisal of a Western text by a former colonial subject may be alternately coded as an act of liberation (or 'writing back') or as a demonstration of postcolonial culture's inherent subordination to the dominant matrix » (38).

${ }^{13}$ Les deux romans sont structurés de la même façon : après l'exposé de la vie quotidienne, suivent le meurtre qui coupe le récit en deux et les conséquences du meurtre. Le meurtre tranche le récit en deux, intervient pour dépecer la matière textuelle comme s'il s'agissait d'un cadavre.
} 
Convergences francophones 6.3 (2020): 41-57

http://mrujs.mtroyal.ca/index.php/cf/index

l'hypotexte (222). ${ }^{14}$ Cette catégorie de texte identifiée par Genette semble en effet pertinente, car Meursault, contre-enquête - véritable texte à double tranchant oscille constamment entre prolongement et antagonisme, angoisse de l'héritage et humble admiration, annihilation du patrimoine et désir d'inscription dans les rangs du grand génie. L'intertextualité assumée par Daoud va plus loin encore, au point d'élaborer ce que je désignerai comme une intertextualité recto-verso. ${ }^{15}$ Dans un acte subversif qui emprunte au genre policier pour combler les blancs laissés par Camus autour du meurtre, Daoud place symboliquement son texte récupératif au verso de celui de Camus. S'il est possible d'imaginer que l'hypotexte autoritaire de Camus s'écrit au recto de la feuille de l'histoire, le verso que vient occuper Daoud en est le reflet inversé qui met constamment en question l'histoire, par exemple en remédiant à ses omissions et en renversant ses hiérarchies, sans jamais nier au textesouche sa valeur de chef-d'œuvre. Ainsi Meursault, contre-enquête se présente comme un reflet difforme, voire une façon de se libérer de l'angoisse de l'héritage et du traumatisme qu'incarne Haroun. ${ }^{16}$

Loin en effet de vouloir s'inscrire dans les rangs de ceux qui souhaiteraient imiter le grand maître, Haroun précise dès le début : "Le meurtrier est devenu célèbre et son histoire est trop bien écrite pour que j'aie dans l'idée de l'imiter. » (Daoud 12). Cette correspondance recto-verso entre les deux textes est rendue encore plus évidente par le fait que les deux livres sont de longueur identique. ${ }^{17}$ En

${ }^{14}$ Donnant plusieurs exemples de «continuations meurtrières », tels que Roland amoureux de Boiardo, Gargantua et Pantagruel de Rabelais ou L'Homme qui était mort de D.H. Lawrence, Genette précise qu' « un vrai créateur ne peut toucher à l'œuvre d'un autre sans y imprimer sa marque. La continuation devient ainsi, dans les meilleurs cas, prétexte à récriture oblique » (223).

${ }^{15}$ Un autre texte qui revêt la forme plutôt littérale de ce type d'intertextualité superposée est le fameux Glas de Derrida qui combine les écrits philosophiques de Hegel (colonne de gauche) et les écrits autobiographiques de Jean Genet (colonne de droite). Dans son livre Ethical issues in Twentieth-century French Fiction: Killing the Other, Colin Davis souligne la spécificité de l'appropriation des textes transgressifs de Genet par Derrida. Les critiques qui ont précédé Derrida (dont Sartre), ont essayé soit de réhabiliter l'œuvre de Genet et d'y trouver une valeur positive, soit de la rejeter en dénonçant son aspect immoral. Le texte de Derrida diffère selon Davis de ces deux tendances car la publication du Glas : " opened up new perspectives in Genet criticism in part through its endeavour to avoid global interpretations and recuperative moral judgements... Derrida focuses on the textual complexity of Genet's writing without attempting to find in it thematic or aesthetic unity » (Davis 158). Meursault, contre-enquête s'inscrit dans cette veine d'hypertextes qui refusent d'assumer un discours explicatif ou moralisateur. À ce propos, voir ci-dessous la partie «'Cela ne voulait rien dire' - contre-enquête de vérité ».

${ }^{16}$ Voir Christiane Chaulet Achour qui compare les réécritures de L'Étranger dans Nedjma de Kateb Yacine et dans Meursault, contre-enquête de Daoud : «Haroun, enfant traumatisé par la violence des aînés, a passé sa vie à faire semblant de vivre, à ne pas se retrouver dans le miroir du pays qu'on lui tendait, à regarder avec curiosité les 'roumis' revenant au pays. » (« Retour Ou Détour Par Camus Dans Le Roman Algérien : Kateb Yacine (1956) / Kamel Daoud (2013)» 3).

17 «The Meursault Investigation is the same length as Camus's The Stranger (33,000 words), and in the perfect middle of the story, Daoud places a murder, just as Camus did before him » (Kaplan 208). Il existe en effet deux éditions de Meursault, contre-enquête - l'une publiée en Algérie en 2013 chez Barzakh et l'autre en France chez Actes Sud en 2014. Ces deux éditions ne sont pas identiques, quelques détails ayant été modifiés dans l'édition française parmi lesquels le plus frappant est le changement du titre de L'Étranger, qui devient L'Autre dans l'édition de 2014. 
Convergences francophones 6.3 (2020): 41-57

http://mrujs.mtroyal.ca/index.php/cf/index

écrivant en même temps à l'encontre et à l'appui du texte de Camus, Daoud se débarrasse du fantôme de l'héritage caractéristique d'une certaine littérature postcoloniale $^{18}$ et transcende du même coup le grand classique qui hante les écrivains algériens, ${ }^{19}$ à travers la mise en scène de l'acte du meurtre. Employé à des fins divergentes, le meurtre s'avère crucial à la fois pour la réflexion métaphysique dans le roman existentialiste et pour la forme propre au roman policier. ${ }^{20}$ Tout comme L'Étranger, le corps textuel du Meursault, contre-enquête est coupé en deux par cet acte transgressif qui permet à Daoud de liquider son patrimoine.

\section{L'Écrivain-tueur}

Meursault, contre-enquête débute par l'épigraphe que Daoud emprunte à E.M. Cioran issu de ses Syllogismes de l'amertume : "L'heure du crime ne sonne pas en même temps pour tous les peuples. Ainsi s'explique la permanence de l'histoire. » (Daoud 7). "L'heure du crime » que Daoud met en exergue pourrait être interprétée de façon littérale, car, alors que Meursault tue l'Arabe à 14 heures par un après-midi ensoleillé dans les années trente, l'heure du crime d'Haroun survient une trentaine d'années plus tard, plus précisément à 2 heures du matin, le 5 juillet 1962 - le jour où l'Algérie acquiert son Indépendance après sept années de lutte armée. La symétrie semble être complète : au meurtre diurne de l'Arabe anonyme sous l'influence oppressive du soleil brûlant correspond le meurtre nocturne du Français sous la lumière faible de la lune. ${ }^{21}$ Le mot histoire dans la deuxième partie de la citation (« ainsi s'explique la permanence de l'histoire »), ne devra pas être compris uniquement comme séquence chronologique d'événements s'échelonnant dans le temps, mais également comme suite d'événements faisant l'objet d'un récit. Dans cette optique, Daoud pose l'équivalence entre le crime et l'écriture dès le début. De surcroît, L'Étranger et Meursault, contre-enquête sont tous deux nés à l'intersection de deux cultures et deux nations, algérienne et française, elles-mêmes liées par un crime inaugural, dont la responsabilité est

\footnotetext{
${ }^{18}$ Voir à ce sujet Bill Ashcroft, Gareth Griffiths, et Helen Tiffin The Empire writes back - theory and practice in post-colonial literatures et Ankhi Mukherjee What is a classic? Postcolonial rewriting and invention of the canon.

19 Sur l'influence de Camus sur les écrivains algériens, voir Christiane Chaulet-Achour, « Fraternités, rivalités, postérités » dans Albert Camus et l'Algérie, Christiane Chaulet-Achour «Camus and Algerian Writers» in Camus' L'Étranger : fifty years on et Martine Mathieu-Job «Comment les écrivains algériens francophones lisent-ils Camus?».

${ }^{20}$ Les fonctions narratives du meurtre ne sont pas les mêmes dans les romans existentialistes et policiers. Alors que le meurtre (tel que le meurtre de l'Arabe par Meursault) permet dans le roman existentialiste d'élaborer une réflexion métaphysique sur la valeur et le sens de la vie, dans le roman policier il sert à des fins plutôt logistiques, mettant en mouvement l'intrigue, suscitant la curiosité ou le suspense, préparant par sa transgression même le rétablissement de l'ordre par l'inspecteur ou le détective. Le roman de Daoud se place à l'intersection de ces deux fonctions du meurtre, car le meurtre commis par Haroun pourrait être qualifié de meurtre existentialiste en même temps qu'il est mobilisé à l'intérieur d'un roman qui emprunte certains de ses codes au roman policier.

${ }^{21}$ « la lune m'a obligé à achever l'œuvre que ton héros avait entamée sous le soleil. A chacun

l'excuse d'un astre et d'une mère » (42).
} 
Convergences francophones 6.3 (2020): 41-57

http://mrujs.mtroyal.ca/index.php/cf/index

unilatérale : celui de la colonisation (1830-1962). La transgression du meurtre est double : d'un côté elle est incarnée par le meurtre diégétique ; de l'autre côté ce meurtre fictif est le résultat de la rencontre entre le colon et le colonisé, qui est rendue possible par la transgression historique et politique - celle de la colonisation de l'Algérie par la France. Or, Haroun ne distingue pas du tout Meursault, le personnage, de l'écrivain, Albert Camus. Il va jusqu'à baptiser l'écrivain de L'Étranger Albert Meursault ${ }^{22}$, et tout au long du livre lui donne de nombreux surnoms qui ont pour effet de souligner ce lien étroit entre l'écrivain et le criminel : « ton héros », « ton écrivain », « le meurtrier », « l'assassin », « un cadavre », « un homme qui sait écrire », « ton Meursault», « l'écrivain tueur». En outre, dans l'imaginaire d'Haroun, l'écriture et le meurtre appartiennent au même champ sémantique : il veut « commettre un livre ${ }^{23}$, ou bien $~ "$ supprimer ${ }^{24}$ le Français.

Cette fusion du personnage et de l'aut(u)eur semble être la réponse directe de la part de Daoud à ceux et celles qui ont accusé Camus-l'écrivain de racisme, sous prétexte que le protagoniste de L'Étranger tue un Arabe anonyme ${ }^{25}$. Ainsi, dans son analyse de Meursault, contre-enquête, Alice Kaplan distingue-t-elle nettement la représentation fictionnelle romanesque, de la rhétorique polémique de l'auteur : « representing a racist society in a novel is not proof of racism » (344). Daoud satirise cette confusion davantage, car dans Meursault, contre-enquête, le meurtre de Moussa n'est pas seulement un mécanisme romanesque qui mobilise l'intrigue, mais aussi un acte extra-diégétique qui assure la renommée de l'écrivain-meurtrier : « S'il n'avait pas tué et écrit, personne ne se souviendrait de lui » (Daoud 88). Un « bon » meurtrier égale donc un bon écrivain, alors que le mot « crime » désigne simultanément le meurtre et sa narrativisation : « Il y en avait des milliers comme lui, à cette époque, mais c'est son talent qui rendit son crime parfait » (74). De plus, l'acte du meurtre est étroitement lié à la création du discours fictif : « Quand on tue,

${ }^{22}$ Il existe, pourtant, une différence majeure entre les éditions algérienne et française. Dans l'édition algérienne le personnage s'appelle Albert Meursault et son livre L'Étranger, ce qui souligne davantage le lien entre le texte de fiction et le contexte colonial historique : « Le titre en était L'Étranger, le nom de l'assassin était écrit en lettres noires et strictes, en haut à droite : Albert Meursault » (Meursault, contre-enquête, Éditions Barzakh, 171). Cependant, dans l'édition Actes Sud l'écrivain s'appelle juste Meursault et son œuvre est intitulé L'Autre, ce qui minimise l'identification explicite de Camus à Meursault : «Le titre en était L'Autre, le nom de l'assassin était écrit en lettres noires et strictes, en haut à droite : Meursault » (Meursault, contre-enquête, Actes Sud, 137).

${ }^{23}$ « Ah, tu sais, moi qui pourtant ne me suis jamais soucié d'écrire un livre, je rêve d'en commettre un » (Daoud 35).

${ }^{24}$ «À chaque élan du désir, je savais que le vivant ne reposait sur rien de dur. Je pouvais le supprimer avec une telle facilité que je ne pouvais l'adorer - ç'aurait été me leurrer » (Daoud 86).

${ }^{25}$ « [Daoud] wanted to mock a literary climate where people have always confused Meursault, who killed an Arab in a book, with the author of that book, Albert Camus. . . What better way than this absurd moniker, 'Albert Meursault', to make the point that Camus is still on trial in Algeria for his intentions - hostage to a fatal confusion of author and character? » (Kaplan 207). Pour en savoir plus sur les critiques qui ont trouvé la reproduction du discours colonial dans les œuvres de Camus, voir : Edward Said, "Camus and the French Imperial Experience» in Culture and Imperialism; Pierre Nora, Les Français d'Algérie; David Carroll, Albert Camus the Algerian: Colonialism, Terrorism, Justice et Conor Cruise O'Brien, Albert Camus : Of Europe and Africa. 
il y a une part de vous qui, immédiatement, se met à échafauder une explication, à fabriquer un alibi, à construire une version des faits qui vous lave les mains alors qu'elles sentent encore la poudre et la sueur » (107). En outre, Haroun ne serait pas tueur s'il n'était pas d'abord lecteur de Camus, ce qui implique de nouveau une forme de complicité forcée. Haroun, lecteur de Camus que traumatise la violence du meurtre en apparence sans motif de l'Arabe, est fait prisonnier de L'Étranger, parce qu'il est en devenu complice malgré lui, et c'est ce traumatisme de la rencontre avec la violence de l'œuvre-source qui a fait de lui un meurtrier. La canonisation de L'Étranger s'articule sur les restes du corps putride du colonisé tué : «Entre les deux temps, mon frère s'est décomposé et le livre a eu le succès que l'on sait » (15). Cette chaîne de transformations et de mises en abyme qui brouillent les limites entre la fiction et la réalité se prolonge lorsque celui qui souhaite réhabiliter la victime devient bourreau à son tour (Haroun assassine le Français). En même temps, le lecteur de L'Étranger (Haroun) se transforme en narrateur de sa propre histoire. Ensuite, le personnage «réel» du fait divers (Meursault) devient l'écrivain fictif dans l'histoire d'Haroun. Enfin, un simple faitdivers, découpé dans des journaux se métamorphose en un des plus grands classiques du $20^{\mathrm{e}}$ siècle. ${ }^{26}$ Ce sont, d'après le narrateur de Daoud, le génie littéraire et un savoir-faire narratif qui contribuent à l'absolution du criminel et qui dissimulent la gravité de son crime ; Haroun précise qu'Albert Meursault «savait raconter, au point qu'il a réussi à faire oublier son crime » (11). Alors que la langue parfaitement ciselée d'Albert Meursault qui selon Haroun choisit « les mots avec l'économie de sa respiration » a servi à camoufler son meurtre, la langue spontanée, familière et dynamique ${ }^{27}$ du narrateur enivré dans Meursault, contre-enquête révèle l'envers du crime (136).

Loin d'éviter le massacre, Haroun procède à une excavation symbolique du corps assassiné qui ne mènera, au terme, qu'à des crimes nouveaux. La narration ne permet pas de mettre fin aux massacres ; bien au contraire, ce sont de nouveaux crimes qui assurent « la permanence de l'histoire » (Daoud 7). Ainsi, en dépit de la tentative d'Haroun de réhabiliter son frère tué, celui-ci restera à jamais hors de notre portée, interdit puisque privé d'accès à la parole et réduit au silence éternel. L'histoire de la victime ne peut être narrée que par l'intermédiaire d'un tiers ${ }^{28}$. Le

\footnotetext{
${ }^{26}$ L'importance du fait divers dans l'œuvre de Camus n'a pas été largement explorée par les critiques (Voir Tran Huy Minh dans Les écrivains et le fait divers, dans le chapitre « La cour d'assises comme laboratoire » 169 - 233 et David H. Walker, « Albert Camus and the fait divers »).

${ }^{27} \mathrm{La}$ narration de Haroun reflète un langage vif et spontané, dans lequel les adresses à l'interlocuteur, que le narrateur tutoie, abondent.

${ }^{28}$ Voir la scène dans Les Justes de Camus, où Stépan raconte à Dora l'exécution de Kaliayev, l'exécution restant hors scène : «Dora : Raconte tout. J'ai le droit de savoir. J'exige que tu racontes. Dans le détail.

Stepan : Je ne saurai pas. Et puis, maintenant, il faut partir.

Dora : Non, tu parleras. Quand l'a-t-on prévenu ? Stepan : À 10 heures du soir. Dora : Quand l'a-ton pendu ? Stepan : À deux heures du matin. Dora : Et pendant quatre heures, il a attendu ?

Stepan : Oui, sans un mot. Et puis, tout s'est précipité. Maintenant, c'est fini. Dora : Quatre heures
} 
Convergences francophones 6.3 (2020): 41-57

http://mrujs.mtroyal.ca/index.php/cf/index

récit sert ainsi de véhicule qui facilite et légitime le meurtre à la fois. Ainsi se produit la dialectique entre la construction et la destruction qui est inhérente à toute écriture-palimpseste : c'est de l'acte suprême de la destruction d'autrui, qu'émane le récit. ${ }^{29}$

\section{« Une histoire coincée entre quelques murs »}

Tout au long du livre, et particulièrement dans l'épisode du meurtre du Français, le discours du narrateur est empreint du vocabulaire du théâtre $:^{30} \ll \mathrm{Je}$ vais te résumer l'histoire avant de te la raconter : un homme qui sait écrire tue un Arabe qui n'a même pas de nom ce jour-là - comme s'il l'avait laissé accroché à un clou en entrant dans le décor» (Daoud 15, je souligne). Sur la scène de ce théâtre, les protagonistes portent des costumes et échangent leurs rôles. Suite à l'Indépendance le colonisé prend la place du colonisateur, Haroun devient Meursault : ${ }^{31}$ « Mais ça, il est vrai, c'était avant que la scène ne tourne sur le moyeu et n'échange les rôles. Avant que je ne réalise à quel point nous étions, lui et moi, les compagnons d'une même cellule dans un huis clos où les corps ne sont que des costumes » (20, je souligne). Le meurtre commis par Meursault à la plage n'est qu'une scène de théâtre inerte : «L'autre balcon invisible de ma tête donne sur la scène de la plage incandescente, la trace impossible du corps de Moussa et sur un soleil figé au-dessus de la tête d'un homme qui tient une cigarette ou un revolver, ${ }^{32}$ je ne sais pas vraiment. J'aperçois la scène de loin » (82, je souligne). A l'instar d'une scène au théâtre, le lieu du crime n'est qu'un fragment de l'espace, délimité par les accessoires du théâtre : «Dans le coin, il y a les pilotis d'un cabanon et, à l'autre bout, le rocher qui clôt cet univers » (82). La mort de Joseph Larquais marque le dénouement de la pièce : "Je trouvais ridicule qu'un homme puisse mourir avec autant de facilité et clore notre histoire avec son affaissement théâtral, presque comique » (86). Le corps de Moussa fait partie de la scénographie : « Mon frère Zoudj, lui, est discrètement retiré de la scène et entreposé je ne sais où » (56).

D'après Barthes, L'Étranger emprunte plusieurs topoï à la mythologie antique et au théâtre classique, ${ }^{33}$ tels que l'ananké et l'inévitabilité du destin prescrit

\footnotetext{
sans parler ? Attends un peu. Comment était-il habillé ? ... Stepan : Laisse, Dora.

Dora : Non, je veux savoir. Sa mort du moins est à moi » (Camus 388 - 389).

${ }^{29}$ Voir Uri Eisenzweig, Le récit impossible.

30 « c'est un peu ma mission : être revendeur d'un silence de coulisses alors que la salle est vide »; « Il ne s'agissait pas seulement d'un cadavre à enterrer, mais d'une scène à ranger, à nettoyer, comme après le dernier acte, au théâtre » (Daoud 11,88).

${ }^{31}$ «L'idée me traversa que je pouvais enfin aller au cinéma ou nager avec une femme. » (Daoud 87)

${ }^{32}$ Allusion notoire au port de la cigarette chez Camus.

${ }^{33}$ « Meursault est charnellement soumis au Soleil, et je crois qu'il faut entendre cette soumission dans un sens à peu près sacral. Tout comme dans les mythologies antiques ou la Phèdre racinienne, le Soleil est ici expérience si profonde du corps, qu'il en devient destin ; il fait l'histoire, et dispose, dans la durée indifférente de Meursault, certains moments générateurs d'actes. Il n'y a aucun des trois épisodes du roman (l'enterrement, la plage, le procès), qui ne soit dominé par cette présence du soleil ; le feu solaire fonctionne ici avec la rigueur même de la Nécessité antique » (Barthes 400).
} 
Convergences francophones 6.3 (2020): 41-57

http://mrujs.mtroyal.ca/index.php/cf/index

par les dieux, la dimension cosmique qui exerce une influence maléfique sur les hommes. ${ }^{34}$ Daoud construit une scène qui ressemble bien davantage au théâtre brechtien : en insistant sur l'artificialité de la mise en scène du meurtre du Français, Haroun brise le quatrième mur et produit par conséquent un effet de distanciation. ${ }^{35}$

Tandis que dans L'Étranger le Soleil oppressif est décrit comme faisant partie du décor naturel, ${ }^{36}$ le clair de lune qui préside à la scène du meurtre commis par Haroun paraît une présence extérieure, simulée et artificielle, étrangère à la réalité fictionnelle. Au premier abord la lune apparaît comme la négation du soleil camusien, comme son côté obscur. ${ }^{37}$ Elle est la «dernière trace pâle du soleil disparu ", et tout comme le soleil dans L'Étranger, elle est personnifiée et animée («[L]la lune regardait elle aussi »). A l'image du lecteur, elle devient complice aussi, en tant que « seul témoin aveugle » du crime (Daoud 96, 86, 90). Toutefois, contrairement au soleil camusien, elle n'est pas menaçante et lointaine, mais palpable et à portée de main tel un accessoire au théâtre, « tellement proche qu'on aurait pu l'atteindre en s'élançant haut vers le ciel», alors que dans l'obscurité de ce décor artificiel il suffisait « d'appuyer sur l'interrupteur pour que le ciel se rallume » $(85,89)$. Toute la nuit « ressemblait à un rideau léger » $(90)$, expression qui évoque le rideau de théâtre. La juxtaposition intertextuelle de la nuit obscure au soleil brillant à son apogée s'étend à l'opposition entre les catégories géopolitiques de l'Orient (soleil levant) et de l'Occident (soleil couchant) qui soustend des crimes eux-mêmes doubles : les meurtres de Moussa et de Joseph et plus d'un siècle de colonisation de l'Algérie par la France.

De plus, le lieu du crime est un espace de la violence historique. La plage comme espace liminal où se croisent le colonisé et le colonisateur venu de l'autre côté de la Méditerranée ${ }^{38}$ est emblématique de la violence colonisatrice. C'est là que Meursault tire cinq fois sur l'Arabe anonyme. Chez Daoud, le temps même

\footnotetext{
${ }^{34}$ Voir aussi André Bernand, La carte du tragique : la géographie dans la tragédie grecque. D'après Bernard, le soleil est un des référents essentiels pour la géographie de la tragédie grecque, dans laquelle tout comme chez Camus, le soleil joue le rôle du témoin du crime, de l'œil omniprésent auquel l'homme ne peut pas échapper : «le soleil, témoin de toutes les actions des hommes, est le grand justicier des fautes humaines, et dans cette mesure est aussi redoutable que la foudre... Dans le pays de la lumière incomparable, l'homme ne peut se résoudre à ne plus voir l'éclat du soleil ... les hommes vivent sous l'œil même du soleil... le héros ne peut manquer d'être aperçu par cet œil qui voit tout... ce guetteur suprême de la conduite humaine » $(151,152)$. Sur le lien entre Camus et l'antiquité voir : Benoît Quinquis, L'Antiquité chez Albert Camus : Caligula, La peste et La chute et Martin Rodan, Camus et l'antiquité.

${ }^{35}$ Voir Bertolt Brecht, « Nouvelle technique d'art dramatique » (1935-41), Écrits sur le théâtre. Paris : L'Arche, 1963 : 330-37.

${ }^{36}$ Nombre de critiques ont commenté l'importance du soleil et de la lumière dans l'œuvre de Camus, dont E. Roblès, La marque du soleil et de la misère ; R. Quilliot, La mer et les prisons ; J.A.G. Tans, La poétique de l'eau et de la lumière d'après l'œuvre d'Albert Camus, Roland Barthes, «'L'Étranger', un roman solaire ».

${ }^{37}$ Cela dit, il semble important de noter les deux symboles présents sur le drapeau algérien : le croissant de lune rouge qui symbolise l'islam et l'étoile.

${ }^{38}$ Haroun décrit la mer méditerranéenne comme suit : « Le ciel était gris et j'avais, à quelques mètres de moi, l'immense, la grande rivale de notre famille, la voleuse d'Arabes et tueuse de maraudeurs en bleu de chauffe. C'était bel et bien le dernier témoin sur la liste de M'ma » (Daoud 54).
} 
devient modulable et recommence artificiellement: "Je devais m'emparer de l'horloge de toutes mes heures vécues, en remonter le mécanisme vers les chiffres du cadran maudit et les faire coïncider avec l'heure exacte de l'assassinat de Moussa : quatorze heures-zoudj. Je me mis à entendre jusqu'au cliquetis de ces rouages reprenant leur tic-tac net et régulier » (88-89) et c'est à ce moment-là que le narrateur (re)naît : "Quant à moi, que te dire ? La vie m'était enfin redonnée même si je devais traîner un nouveau cadavre. Du moins, me disais-je, ce n'était pas le mien, mais celui d'un inconnu » (90). Le meurtre commis par Haroun a donc un rôle régressif et inaugural. Une fois que Joseph Larquais est tué, le temps recommence et le narrateur régresse vers un stade primitif, pré-linguistique : « Ce furent des jours sans noms ni langage, je percevais les êtres et les arbres autrement, sous un angle inattendu, au-delà de leur dénomination usuelle, revenant à la sensation primitive »(135-136). Avec ce patrimoinicide symbolique, l'histoire (dans les deux sens du mot) s'efface et Haroun retrouve la page vierge. Le meurtre du Français coïncidant avec l'Indépendance de l'Algérie, Haroun reconquiert simultanément sa page et sa plage. Il se rapproprie sa terre et la plage qui a donné lieu au meurtre de son frère et retrouve ainsi sa place sous le soleil camusien.

Suite au meurtre du Français, la présence invasive du texte-souche fusionne peu à peu avec Meursault, contre-enquête, et le dialogue intertextuel disparaît pour laisser place à un monologue libérateur, où le texte se transforme en un espace où le soi se construit un semblant de souveraineté. Dans une fusion entre le bourreau et la victime, entre l'hypotexte et l'hypertexte, entre la réalité et la fiction, le personnage littéraire et l'écrivain, à la suite d'un acte symbolique de patrimoinicide, Haroun-CEdipe est libre de raconter son histoire. Le narrateur s'approprie une histoire qu'il ne désigne plus à l'étudiant qui l'écoute comme le fait de «ton héros », mais bien comme « mon histoire à moi » dont le narrateur est le maître absolu, voire même tyrannique (114). Ce narrateur autoritaire est sans merci. Ses personnages sont des marionnettes qu'il manipule à son gré, à l'instar des dieux dans la tragédie antique : «Il [le Français] était là, coincé entre deux histoires et quelques murs, avec pour seule issue mon histoire à moi qui ne lui laissait aucune chance » (114). Le Français n'est donc qu'un outil, ou même un obstacle narratologique « coincé entre deux histoires » : son existence est purement textuelle. Suite à ce démantèlement de « quelques murs » (le célèbre quatrième mur entre eux) qui ont coincé la victime, les propriétés textuelles sont révélées et le lecteur ne participe plus à la suspension volontaire de l'incrédulité. Libéré de son prédécesseur, le narrateur arrange le décor à son gré, démonte et recompose la scène du meurtre, en réduisant celle-ci à un ensemble d'accessoires au théâtre :

balayer le sable de la plage, enfouir le corps dans un pli froncé de l'horizon, repousser le fameux rocher des deux Arabes et le balancer derrière la colline, dissoudre l'arme comme s'il s'agissait d'une écume, appuyer sur l'interrupteur pour que le ciel se rallume et la mer reprenne ses halètements et, enfin, remonter vers le cabanon pour rejoindre les personnages figés de cette histoire (89) 
Sans le narrateur qui donne le (dernier) souffle de vie à ses personnages, ceux-ci resteraient figés, inanimés, comme des cadavres. Haroun est finalement le maître de l'histoire qu'il raconte puisqu'il tire les ficelles de ses personnagesmarionnettes. Le meurtre commis par Haroun qui lui permet d'assumer sa voix, coïncide avec la libération de la terre algérienne face aux oppresseurs français. Grâce à la transgression meurtrière, Haroun parvient à occuper un espace, à marquer son territoire. Libéré du poids de la victimisation et de la marginalité, le narrateur découvre avec étonnement l'étendue d'un espace (en même temps littéraire et géopolitique) qui lui appartient et au sein duquel il inscrit sa voix :

J'ai encore une fois été surpris par l'immensité de la cour où je venais d'exécuter un inconnu. Comme si les perspectives se dégageaient et que je pouvais enfin respirer. Alors que jusque-là, j'avais toujours vécu enfermé dans le périmètre tracé par la mort de Moussa et la surveillance de ma mère, je me vis debout, au cœur d'un territoire déployé à la mesure de toute la terre nocturne et offerte cette nuit (Daoud 108)

En soulignant de la sorte l'artifice de la mise en scène ainsi que les aspects métatextuels de la genèse de l'histoire d'Haroun, Daoud brise le quatrième mur et fait douter le lecteur de la vraisemblance du contenu romanesque. Par là même, l'écrivain retire le texte de Camus d'un panthéon intouchable, détrônant le grand classique et le réduisant au statut d'un objet que l'on peut manier ou disposer à son gré.

\section{Un texte étranger}

L'Étranger serait un texte immuable, pétrifié, achevé - en somme, un texte étranger et opaque, protégé par une vitre impénétrable : « c'est une scène immuable contre laquelle je bute comme une mouche sur une vitre. Impossible d'y pénétrer. Je ne peux y poser le pied pour courir sur le sable et changer l'ordre des choses » (107). Dans son livre Ethical Issues in Twentieth-Century Fiction: Killing the Other, Colin Davis propose une solution possible à l'énigme que pose le meurtre de l'Arabe par Meursault dans L'Étranger. Selon Davis, le meurtre est le résultat d'une réciprocité entre la communauté et l'exclusion qui est au centre de nombreuses auvres de Camus : "The murder of the Arab represents the most overtly violent, litteral attempt to commit altericide in a set of texts which repeatedly dramatize the suppression and reappearance of the Other » (66). Cette dialectique entre inclusion et exclusion se reproduit au niveau de l'incorporation directe de phrases de L'Étranger dans Meursault, contre-enquête, où elles paraissent en italique. ${ }^{39}$ Sans constituer un hommage, ce geste met en relief l'étrangeté de l'hypotexte. Les mots en italique se greffent sur le texte de Daoud comme un corps détaché qui ne lui appartient pas. L'Étranger est une œuvre figée

\footnotetext{
${ }^{39}$ À titre d'exemple : « Tels des spectres discrets et muets, ils (les Français) nous regardaient, nous les Arabes, en silence, ni plus ni moins que si nous étions des pierres ou des arbres morts. »; « Devant moi, tout était ombre et chaque objet, chaque angle, toutes les courbes se dessinaient avec une confusion insultante pour la raison » (Daoud 21, 94).
} 
dans le temps et dans l'espace mais qui refuse de fournir une signification stable et unique. Contrairement à L'Étranger, Meursault, contre-enquête est un texte dynamique, qui s'inscrit dans une tradition d'oralité et d'inachèvement. Un autre livre avec un L majuscule qui partage ce statut sacré et autoritaire est, dans l'imaginaire du narrateur, le Coran, « leur livre à eux » tout aussi ambigu : « Je feuillette parfois leur livre à eux, Le Livre, et j'y retrouve d'étranges redondances, des répétitions, des jérémiades, des menaces et des rêveries » (Daoud 81). De plus, l'aspect sacré du texte canonique de Camus est davantage souligné par le fait que la femme qui révèle $L$ 'Étranger à Haroun s'appelle Meriem, qui porte le nom, dans sa version arabe, de la mère de Jésus qui apparaît dans le Nouveau Testament comme dans le Coran : « Moi, je connais ce livre [L'Étranger] par cœur, je peux te le réciter comme le Coran. ». L'Étranger est l'histoire du conflit entre l'homme et le soleil, comme c'est, selon le narrateur, le cas dans le Coran : «J'ai le sentiment qu'il ne s'agit pas d'un livre mais d'une dispute entre un ciel et une créature » (75).

A l'instar de L'Étranger, Daoud a soigneusement divisé son livre en deux parties. La transformation d'Haroun a lieu une fois qu'il a découvert le livre d'Albert Meursault; cette découverte marque le moment où il se transforme de sujet colonisé, en un double du colonisateur. Ainsi le moment charnière de Meursault, contre-enquête est-ce moins le meurtre du Français que la découverte que fait Haroun du texte de L'Étranger (L'Autre dans l'édition française), soit la découverte de l'altérité textuelle. Le texte autre est traité comme un corps étranger, un intrus qui vient se mêler à l'histoire d'Haroun.

De surcroît, le livre de Camus est fétichisé sous la plume de Daoud. Le narrateur se moque avec ironie de l'idée reçue selon laquelle les œuvres canoniques auraient découvert une vérité universelle. La prose de Camus est représentée comme quelque chose de pur, de clair et d'exact qui aurait une valeur transcendantale inhérente. L'écrivain de L'Étranger est comparé à un alchimistemathématicien qui connaît le bon dosage, alors que la violence extrême se transforme en pure poésie sous sa plume-baguette magique :

Il écrit si bien que ses mots paraissent des pierres taillées par l'exactitude même. C'était quelqu'un de très sévère avec les nuances, ton héros, il les obligeait presque à être des mathématiques. D'infinis calculs à base de pierres et de minéraux. As-tu vu sa façon d'écrire ? Il semble utiliser l'art du poème pour parler d'un coup de feu! Son monde est propre, ciselé par la clarté matinale, précis, net, tracé à coups d'arômes et d'horizons (Daoud 12, je souligne).

Daoud va encore plus loin en satirisant le topos du génie littéraire dont la prose serait parsemée de pierres précieuses. Eternelle et de parfait calibre, elle permet à l'écrivain-magicien de transformer la réalité banale en " diamants » : "Personne, même après l'Indépendance, n'a cherché à connaître le nom de la victime, son adresse, ses ancêtres, ses enfants éventuels. Personne. Tous sont restés la bouche ouverte sur cette langue parfaite qui donne à l'air des angles de diamant » (14). Le talent du créateur du chef-d'œuvre est hyperbolisé, tandis que l'admiration se transforme en idolâtrie : «Je reconnais à ton héros le talent d'inventer une tragédie 
à partir d'un bout de journal et de raviver l'esprit fou d'un empereur à partir d'un incendie » (72). C'est pour Daoud une autre manière de poser un équivalent entre le génie littéraire et la violence de l'écrit, alors que tout crime est pardonné, du moment qu'il est décrit avec de la virtuosité propre au grand génie.

\section{‘Cela ne voulait rien dire' : contre-enquête de vérité}

Le lecteur/interlocuteur d'Haroun assume le rôle de l'inspecteur du roman policier ${ }^{40}$ qui est en quête de vérité derrière le mystère du crime. Toutefois, contrairement aux romans policiers à énigme traditionnels, où le dénouement aurait résulté en une explication rationnelle de l'acte transgressif, la réponse échappe constamment à l'enquêteur. Le lecteur/interlocuteur curieux, ${ }^{41}$ désireux de connaître les réponses aux énigmes posées par le texte de Camus, reste en mal d'explications :

D'ailleurs, c'est toi qui es venu à moi - je me demande bien comment tu as pu remonter jusqu'à nous! Tu es là parce que tu crois, comme moi autrefois, pouvoir retrouver Moussa ou son corps, identifier les lieux du meurtre et aller claironner ta découverte au monde entier. Je te comprends. Toi, tu veux retrouver un cadavre, alors que moi, je cherche à m'en débarrasser (56)

Le sens de l'histoire se dérobe constamment à l'emprise de "l'inspecteur universitaire ». En dépit de toute tentative de compréhension, le texte demeure essentiellement l'Autre et étranger « Un point me taraude en particulier : comment mon frère s'est-il retrouvé sur cette plage ? On ne le saura jamais. Ce détail est un incommensurable mystère et donne le vertige, quand on se demande ensuite comment un homme peut perdre son prénom, puis sa vie, puis son propre cadavre en une seule journée » (71). L'Étranger refuse toute interprétation unique et fixe :

Je ne cesse de me demander, encore et encore : mais pourquoi donc Moussa, ce jour-là, se trouvait-il sur cette plage ? Je ne sais pas... Relis le paragraphe dans le livre. Lui-même admet s'être un peu perdu pour tomber presque par hasard sur les deux Arabes... Pourquoi est-il venu sur cette plage ce jour-là justement ? Ce qui est inexplicable, ce n'est pas uniquement le meurtre, mais aussi la vie de cet homme (73)

En refusant de fournir une explication et ainsi aller à l'encontre du conformisme du lecteur, l'altérité du texte de Camus résiste à toute tentative de domestication. En refusant de donner un sens unique, d'expliquer l'œuvre de Camus, ou d'adopter une attitude moralisatrice, Daoud reprend l'ambiguïté de L'Étranger. En parlant de l'appropriation du texte de Genet par Derrida, Davis écrit : «Both appropriation and repudiation ... are strategies of non-reading, forms of resistance to the potent force of Genet's writing » (158). Daoud se rapproche de la démarche intertextuelle de Derrida dans Glas qui «offers no criteria for arresting the dissemination of meaning; and by showing how it is never possible to say everything about the text,

${ }^{40}$ Haroun lui-même appelle son interlocuteur « monsieur l'inspecteur universitaire » (Daoud 30).

${ }^{41}$ « J'attendais un petit curieux comme toi pour pouvoir enfin la [l'histoire] raconter » (Daoud 64). 
he conspicuously fails to say anything » (159). En refusant d'expliquer le sens du texte de Camus, Daoud maintient l'altérité de l'œuvre qui ne saurait être réduite à une interprétation singulière, alors que la relation entre le meurtrier et sa victime se reflète dans celle qui unit le texte et le lecteur/enquêteur : « as the text's Other, the reader must be denied the freedom of response or interpretation which might rob the text of its self-possession. The text, then is engaged in the same struggle for dominance with the reader as the murderer confronted with the loathed and desired Other » (177). En même temps, le texte de Daoud refuse d'adopter une position moralisatrice ou polémique univoque. Bien que Daoud emploie la structure du roman policier, dans lequel l'objectif principal de la narration serait de dévoiler le mystère qui dissimule les mobiles et l'identité de l'auteur du meurtre, Meursault, contre-enquête ne parvient pas à restaurer l'ordre brisé. Le crime reste inexpliqué, et le sens du texte qui «ne voulait rien dire » nous échappe. ${ }^{42}$

Pour conclure, le texte de Daoud ne se montre pas comme la négation complète de L'Étranger, mais plutôt sa contre-enquête, soit une histoire qui remonte à l'envers à partir de la victime. Daoud n'offre aucune explication rationnelle ni résolution définitive à l'énigme du meurtre de l'Arabe, ce qui déstabilise les lecteurs et les empêche toute prise de position conformiste qui faciliterait un retour à l'ordre. Au lieu d'aboutir à une réponse qui aurait clôturé le roman policier traditionnel, le lecteur ne retrouve rien d'autre qu'un narrateur mythomane et logorrhéique en train de regagner son espace littéraire et géopolitique. C'est en se servant du topos de l'enquête policière que Daoud attaque cette œuvre canonique de Camus et inaugure ainsi une certaine intertextualité antagoniste, qui sans éliminer l'œuvre de Camus, la remet en mouvement en exposant son étrangeté.

\footnotetext{
${ }^{42}$ Camus note dans ses Carnets de 1938 que l'œuvre littéraire doit « dire moins » : « au lieu de donner toute l'expérience dans le papier à dentelles d'une littérature d'explication, accepte de n'être qu'un morceau taillé dans l'expérience, une facette du diamant où l'éclat intérieur se résume sans se limiter » (1015).
} 


\section{Bibliographie}

Ashcroft, Bill. The Empire Writes Back: Theory and Practice in Post-Colonial Literatures. London New York : Routledge, 1989.

Assia, Belhabib. «Le mal de mère. Le sanctuaire de la restitution dans Meursault contre-enquête de Kamel Daoud. » Babel 36.1 (2017) : 119-127.

Bakhtin, Mikhaïl Mikhaïlovitch. Rabelais and His World. Bloomington: Indiana UP, 2009.

Barthes, Roland. Euvres Complètes. Paris : Seuil, 1993.

Bernand, André. La Carte Du Tragique : La Géographie Dans La Tragédie Grecque. Paris : Editions du Centre national de la recherche scientifique, 1985.

Brecht, Bertolt et Jean Tailleur. Écrits sur le théâtre. Paris : L'Arche, 1972.

Brozgal, Lia. "The Critical Pulse of the Contre-Enquête: Kamel Daoud on the Maghrebi Novel in French. " Contemporary French and Francophone Studies 20.1 (2016): 37-46.

Camus, Albert et Roger Quilliot. Théâtre, Récits, Nouvelles. Paris : Gallimard, 1962.

Carroll, David. Albert Camus, the Algerian: Colonialism, Terrorism, Justice. New York: Columbia University Press, 2007.

Chaulet-Achour, Christiane. Albert Camus \& l'Algérie : fraternités et tensions. Alger : Éditions Barzakh, 2004.

---. « Retour Ou Détour Par Camus Dans Le Roman Algérien : Kateb Yacine (1956) /Kamel Daoud (2013). »Christiane Chaulet Achour: le Site Officiel, Dec. 2015, christianeachour.net/images/data/telechargements/2015/A295.pdf.

Daoud, Kamel. Meursault, contre-enquête. Algers: Barzakh, 2013.

---. Meursault, contre-enquête. Arles : Actes Sud, 2016.

David H. Walker. «Albert Camus and the fait divers. » French Cultural Studies 3.7 (1992): 1-15.

Davis, Colin. Ethical Issues in Twentieth-Century French Fiction: Killing the Other. Basingstoke New York: Macmillan St. Martin's Press, 2000.

Eisenzweig, Uri. Autopsies Du Roman Policier. Paris : UGE, 1983.

---. Le récit impossible : forme et sens du roman policier. Paris : Bourgois, 1986.

Genette, Gérard. Palimpsestes : La Littérature Au Second Degré. Paris : Seuil, 1982.

Gorrara, Claire. «French Crime Fiction : From Genre Mineur to Patrimoine Culturel. » French Studies 61.2 (2007): 209-214.

Isaac, Jeffrey C. « Camus on Trial. » Dissent, 63.1 (2016): 145-150.

Kaplan, Alice Yaeger. Looking for The Stranger: Albert Camus and the Life of a Literary Classic. Chicago: The University of Chicago Press, 2016.

King, Adele. Camus' L'Étranger: Fifty Years On. Basingstoke: Macmillan, 1992.

Mathieu-Job, Martine. «Comment les écrivains algériens francophones lisent-ils Camus ? » Revue d'histoire littéraire de la France 113.4 (2013): 893.

Mukherjee, Ankhi. What Is a Classic? Postcolonial Rewriting and Invention of the Canon. Stanford : Stanford UP, 2015. 
Nora, Pierre. Les Français d'Algérie: édition revue et augmentée. Christian Bourgeois éditeur, 2012.

---. Les Lieux De Mémoire. Paris: Gallimard, 1984.

O'Brien, Conor Cruise. Albert Camus of Europe and Africa. New York: Viking Press, 1970.

Quilliot, Roger. La Mer Et Les Prisons Essais Sur Albert Camus. Paris : Gallimard, 1970.

Quinquis, Benoit, and Pascal David. L'antiquité chez Albert Camus : Caligula, La Peste et La Chute. Paris : L'Harmattan, 2014.

Roblès, Emmanuel. « La Marque Du Soleil Et De La Misère. » Camus. Ed. Rene Marill Albérès. Paris : Hachette, 1964.

Rodan, Martin. Camus et l'antiquité. Pieterlen: Lang, Peter Bern, 2014.

Roseau, Katherine. « The Meursault Investigation by Kamel Daoud. » Shofar: An Interdisciplinary Journal of Jewish Studies, 34.3 (2016): 117-119.

Saïd, Edward W. Culture and Imperialism. New York : Knopf, 1993.

Tans, J.A.G. «La poétique de l'eau et de la lumière d'après l'œuvre d'Albert Camus. » Style et Littérature. Ed. Pierre Louis Roger Guiraud, Paul Zumthor, and A. Kibédi Varga. La Haye : Van Goor Zonen, 1962. pp.75-95.

Tran Huy, Minh. Les écrivains et le fait divers : une autre histoire de la littérature. Paris: Flammarion, 2017. 